\title{
Do Chronic Obstructive Pulmonary Diseases (COPD) Self-Management Interventions Consider Health Literacy and Patient Activation? A Systematic Review
}

\author{
Uday Narayan Yadav $^{1, *}$, Jane Lloyd ${ }^{1}\left(\mathbb{D}\right.$, Hassan Hosseinzadeh ${ }^{2}\left(\mathbb{0}\right.$, Kedar Prasad Baral $^{3}$ and \\ Mark Fort Harris 1 \\ 1 Centre for Primary Health Care and Equity, UNSW, Sydney NSW-2052, Australia; j.lloyd@unsw.edu.au (J.L.); \\ m.f.harris@unsw.edu.au (M.F.H.) \\ 2 School of Health \& Society, University of Wollongong, Wollongong NSW-2522, Australia; \\ hassanh@uow.edu.au \\ 3 School of Public Health, Patan Academy of Health Sciences, Kathmandu-26500, Nepal; \\ kedarbaral@pahs.edu.np \\ * Correspondence: u.yadav@unsw.edu.au or unyadav1@gmail.com, Tel.: +61-040-584-6602
}

Received: 23 December 2019; Accepted: 26 February 2020; Published: 28 February 2020

\begin{abstract}
Self-management (SM) includes activities that patients initiate and perform in the interest of controlling their disease and maintaining good health and well-being. This review examines the health literacy and patient activation elements of self-management interventions for Chronic Obstructive Pulmonary Diseases (COPD) patients. We investigated the effects of the intervention on health-related quality of life, self-efficacy, depression, and anxiety among people with COPD. We conducted a systematic review of studies evaluating the efficacy of self-management interventions among COPD patients that also included health literacy or patient activation as keywords. Four electronic databases Medline, EMBASE, PsycINFO, and Google Scholar, were searched to identify eligible studies. These studies were screened against predetermined inclusion criteria. Data were extracted according to the review questions. Twenty-seven studies met the criteria for inclusion. All of the included studies incorporated health literacy components and focused on COPD and self-management skills. Three studies measured health literacy; two showed improvements in disease knowledge, and one reported a significant change in health-related behaviors. Seventeen studies aimed to build and measured self-efficacy, but none measured patient activation. Eleven studies with multicomponent interventions showed an improvement in quality of life. Six studies that focused on specific behavioral changes with frequent counseling and monitoring demonstrated improvement in self-efficacy. Two interventions that used psychosocial counseling and patient empowerment methods showed improvement in anxiety and depression. Most self-management interventions did not measure health literacy or patient activation as an outcome. Successful interventions were multicomponent and comprehensive in addressing self-management. There is a need to evaluate the impact of comprehensive self-management interventions that address and measure both health literacy and patient activation on health outcomes for COPD patients.
\end{abstract}

Keywords: Chronic Obstructive Pulmonary Diseases; health literacy; patient activation; self-management

\section{Introduction}

Chronic Obstructive Pulmonary Disease (COPD) is a common, preventable, and treatable disease that is characterized by persistent respiratory symptoms and airflow limitation [1]. The treatment and management of COPD is a significant challenge for health systems worldwide [2]. People suffering from COPD often have worsening symptoms, including breathlessness, which requires self-management 
skills and knowledge. Self-management programs have been demonstrated to slow down the worsening symptoms, prevent exacerbations, and improve quality of life [3]. Self-management behaviors are the practices that patients initiate in the interest of controlling their own disease and maintaining good health and well-being [4]. For COPD, they primarily involve early self-recognition and early self-initiation of treatment for exacerbation, compliance with medication (including immunization), coping with breathlessness, quitting smoking, regular physical exercise, and eating a healthy diet [5]. Systematic reviews suggest that COPD self-management interventions (SMIs) improve health-related quality of life and reduced emergency department visits [6-8].

Health literacy (HL) and patient activation (PA) play key roles in self-management interventions. HL helps patients to develop a "skillset" to better manage their health, while PA develops a "mindset" that helps them "to change the lifestyle behavior" [9]. Interventions aimed at using both HL and PA can greatly benefit COPD patients [9]. Health literacy is defined as "the degree to which individuals can obtain, process, and understand the basic health information and services they need to make appropriate health decisions" $[8,10]$ and, "patient activation" refers to the "knowledge, skills and confidence of a person in managing their own health and care" [11].

Addressing health literacy in self-management interventions has been shown to improve individual decisions and actions $[12,13]$ in the area of smoking, nutrition, alcohol, physical activity, and weight control among people with chronic diseases [14]. Similarly, emerging research shows that patient activation improves patient engagement in self-care practices [15] and healthy lifestyle behavior-change programs [16,17]. This review was undertaken to examine health literacy and patient activation in COPD self-management interventions. This systematic review aimed to provide a broad, overarching synthesis of the existing evidence to inform policy, research, and practice in regard to the position of $\mathrm{HL}$ and PA in self-management interventions for COPD.

\section{Research Question}

Have COPD self-management interventions included health literacy and patient activation components, and have they measured improvements in health literacy and patient activation?

What are the effects of the intervention on health-related quality of life, self-efficacy, depression, and anxiety among people with COPD in self-management interventions (SMIs)?

\section{Methods}

The methodology was guided by the Preferred Reporting Items for Systematic reviews and Meta-Analyses (PRISMA) [18].

\subsection{Search Strategy}

Four well-known electronic databases, Medline, EMBASE, PsycINFO, and Google Scholar, were searched to find eligible RCTs published between 1 January 2008 and 9 December 2019. Combinations of Medical Subject Headings (MeSH) terms, using "OR" and "AND", were used to operate the electronic databases. To be selected, papers had to have self-management and either health literacy or patient activation search terms, as outlined in the Box 1 below. 
Box 1. List of search terms used in conducting systematic review.

\begin{tabular}{|c|}
\hline $\begin{array}{l}\text { Obstructive Pulmonary Disease", and "COPD". } \\
\text { AND } \\
\text { EITHER } \\
\text { Health literacy: The search terms included any of the following: "Health Literacy", "Functional health literacy", } \\
\text { "Interactive health literacy", "Knowledge on health", "Reading and writing", "Literacy level", "Comprehensive } \\
\text { health knowledge", "health information" and "health promotion", "Chronic Obstructive Pulmonary Disease", } \\
\text { and "COPD". } \\
\text { OR } \\
\text { Patient activation: The search terms included any of the following: "Patient activation", "Patient Activation } \\
\text { Measure", "Personalized support", "Motivation", Knowledge", "Skills", "Confidence", "Empowerment", } \\
\text { "Chronic Obstructive Pulmonary Disease", and "COPD". }\end{array}$ \\
\hline
\end{tabular}

\subsection{Inclusion Criteria}

Papers should be RCTs or comparative studies in the English language, available in full text, and aimed at the self-management of COPD. Participants had to be diagnosed with COPD. The intervention had to include elements which addressed either HL or PA. Primary outcome measures had to include quality of life (QOL), anxiety and depression, self-efficacy, or the measurement of health literacy and patient activation.

\subsection{Exclusion Criteria}

Conferences abstracts, posters, studies with an intervention period of less than six months, published protocols, studies with patients with cancer as co-morbidity, telehealth interventions, drug trials, and studies reported in other than English language were excluded from this study.

\subsection{Data Extraction}

Initially, one author (U.N.Y.) screened all titles and excluded articles that were irrelevant under the supervision of MFH. After that, five authors (U.N.Y., M.F.H., H.H., J.L. and K.P.B.) used a standardized form based on eligibility criteria to independently review full-text articles in line with the Preferred Reporting Items for Systematic reviews. Finally, MFH separately examined the full-text articles meeting eligibility and exclusion criteria. There were few discrepancies between U.N.Y. and M.F.H. in the excluded data that were resolved in consensus discussion between the two reviewers. A vote of the majority was used to address disagreements during the review of full texts.

\section{Results}

Initially, 481 potentially relevant articles were identified. Twenty-seven studies met the inclusion criteria. Our screening process is depicted in Figure 1.

\subsection{Health Literacy and Patient-Activation Activities}

\subsubsection{Health Literacy}

All twenty-seven studies included in our review had interventions that address health literacy. This was provided through educational materials (covered information on COPD, self-management skills and COPD medications, breathing techniques, maintaining healthy lifestyle, managing stress and anxiety, inhalation instructions, etc.) and improving clinical communication between health providers and patients. Three [20-22] studies specifically measured health literacy in the form of COPD knowledge and self-management skills and behavior. Of these three studies, two [20,22] showed improvements in disease knowledge, and one study [21] reported a significant change in health-related behaviors. 


\subsubsection{Patient Activation}

Eighteen studies [20,22-38] focused on building self-efficacy, but none on building the overall confidence necessary to activate patients to engage in self-management of behaviors. None of the studies in this review included patient activation and measurement.
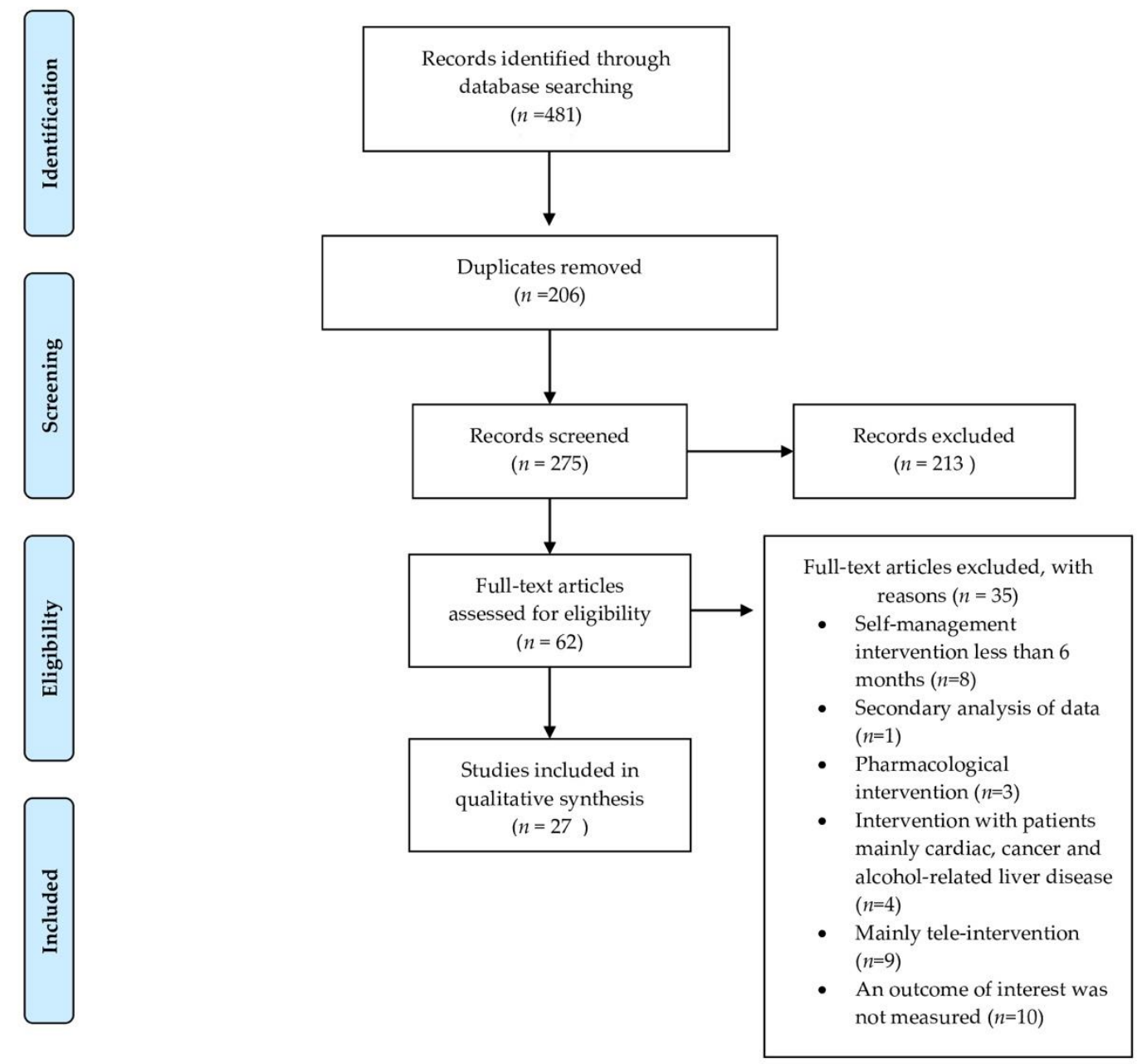

Figure 1. Flow diagram of screening process.

\subsection{Outcomes of Self-Management Intervention}

\subsubsection{Quality of Life}

Health-related quality of life was assessed by twenty-five studies [4,7,20-32,34-43]. Eleven studies $[7,20,22-26,29,31,37,38]$ reported a significantly higher quality of life in the intervention groups compared to the groups with usual treatment. Of these eleven studies that demonstrated improvement in quality of life, ten studies $[20,22-26,29,31,37,38]$ included activities targeted to HL and PA, while one [7] study used HL but did not report on PA. All eleven studies involved were found to be delivering multicomponent self-management interventions (SMIs) programs, whereby five [20,22, $23,31,37]$ were delivered by multidisciplinary health teams, five by respiratory nurses $[7,25,26,29,38]$, and one [24] by lay tutors. The duration of the interventions varied from six months to twenty-four months. In these studies, health-related quality of life was assessed by using a variety of instruments, including EuroQol-5 Dimension (EQ-5D-3L), SF-36 scale, St.-George's Respiratory Questionnaire (SGRQ-C), COPD Assessment Test (CAT), Clinical COPD Questionnaire (CCQ), and Short Form Chronic Respiratory Disease Questioner (CRQ-SF). 


\subsubsection{Self-Efficacy}

The effects on self-efficacy were measured in fifteen studies [20,22,23,25,27,29,30,32-35,38-41]. Six studies $[20,24,27,33,37,44]$ showed significant improvement in self-efficacy. These involved motivational counselling to encourage participants to set short-term behavioral goals required for self-management of COPD. The interventions were often delivered by health professionals, mainly nurses, physiotherapists, and primary care providers; the exception being the two $[24,33]$ studies that delivered the interventions through trained lay tutors. However, none of these studies provided information on the frequency of the motivational sessions.

\subsubsection{Anxiety and Depression}

Eleven studies $[4,20,24-26,31,32,34,36,39,42]$ measured anxiety and depression as an outcome. Of these, nine $[4,20,25,26,32,34,36,39,42]$ did not show a change in anxiety and depression. Only two interventions [24,31] showed improvement in anxiety and depression scores over the period of the intervention. Of these two interventions, one was delivered by trained lay tutors [24] and another [31] by a primary care team. These studies sought to develop patient empowerment and provided psychosocial counselling.

\section{Discussion}

Despite a wealth of evidence showing beneficial outcomes of COPD self-management programs, substantial gaps remained in the evidence base. To the best of our knowledge, this is the first review to analyze the inclusion of health literacy and patient activation elements in COPD self-management interventions. All the included studies (Table 1) evaluated interventions that aimed to address health literacy to some degree (although HL was not necessarily comprehensively addressed). Three studies [20-22] measured disease knowledge as an outcome. None of the authors measured participants' abilities to read, listen, communicate, and understand the provided information, including health promotion, disease prevention, and the navigation of available services. Although many studies addressed and measured self-efficacy, none specifically developed activities designed to activate patients or measured patient activation as an outcome measure. This suggests the need for their role in self-management for long-term conditions, such as Chronic Obstructive Pulmonary Disease to be further explored and evaluated.

In more than a third of the included studies, self-management interventions improved the quality of life of COPD patients. More importantly, QOL improvements were seen majorly in those interventions that addressed both HL and PA (10 out of 11 interventions that improved QOL) to some degree and offered a comprehensive package of self-management components (individual tailored education sessions on disease and self-management, goal-setting and coping strategies, social support, physical activity, improving confidence, etc.). In line with our findings, other reviews have suggested that multicomponent self-management interventions (SMIs) are significantly effective in improving HRQOL [3,6,45]. However, our finding shows marked variation in the measures of quality of life. This heterogeneity prevented meaningful meta-analysis. Ferrone et al. [46] have suggested the use of a single instrument in future research (i.e., using the Clinical COPD Questionnaire (CCQ) - a 10-item, health-related quality-of-life questionnaire). As reported in another review, we found most studies used generic HRQOL measures (i.e., EQ5D and SF scales), and these reported insignificant differences in quality of life [46]. Overall, our finding shows that multicomponent self-management programs having both HL and PA are more likely to yield promising improvements in QOL. 
Table 1. Included studies.

\begin{tabular}{|c|c|c|c|c|c|c|c|}
\hline Study ID & Intervention & Participants Characteristics & Follow-Up & $\begin{array}{c}\text { Activities } \\
\text { Targeted at } \\
\text { Health Literacy }\end{array}$ & $\begin{array}{c}\text { Activities } \\
\text { Targeted to } \\
\text { Activate Patients } \\
\text { in any Form } \\
\end{array}$ & Outcomes & Findings \\
\hline $\begin{array}{l}\text { Chavannes et al. } \\
\text { (2009) [23] }\end{array}$ & $\begin{array}{l}\text { Integrated Disease Management delivered by two } \\
\text { specialized physiotherapists, a respiratory } \\
\text { nurse, a physician assistant, a dietician, a pharmacist, } \\
\text { and a supervising primary care physician. } \\
\text { Intervention includes the following: } \\
\text { rapid action plans for exacerbations, personalized } \\
\text { physical activity training program (at least three } \\
\text { sessions of at least } 40 \text { min of physical activity per week } \\
\text { over three months) and continuous self-management } \\
\text { education including personal goal-setting } \\
\text { by motivational interviewing techniques. }\end{array}$ & $\begin{array}{c}\text { Age } \mathrm{I} / \mathrm{C}: 64 / 63 \text { years } \\
\text { Gender }(n=\mathrm{M} / \mathrm{F}): 59 / 67 \\
\text { Setting: outpatients } \\
\text { Mean \% predicted } \mathrm{FEV}, \mathrm{I} / \mathrm{C}=62 \pm 19 / 66 \pm 16 \\
\text { Dyspnea }(\%) \mathrm{I} / \mathrm{C}=36 / 32 \\
\text { COPD exacerbation } n \\
(\%) \mathrm{I} / \mathrm{C}=\text { Not reported }\end{array}$ & One year & $\begin{array}{l}\text { Education on } \\
\text { disease and } \\
\text { self-management } \\
\text { skills }\end{array}$ & $\begin{array}{l}\text { Motivational } \\
\text { interview }\end{array}$ & $\begin{array}{l}\text { Health-related } \\
\text { quality of life } \\
\text { (HRQOL) }\end{array}$ & $\begin{array}{l}\text { Improvement in } \\
\text { quality of life. }\end{array}$ \\
\hline $\begin{array}{c}\text { Effing et al. (2011) } \\
\text { [39] }\end{array}$ & $\begin{array}{l}\text { Training by physiotherapist } \\
\text { Intervention includes the following: three-session } \\
\text { course (11 h in total) on knowledge about COPD and } \\
\text { exercises training for } 11 \text { months }\end{array}$ & $\begin{array}{c}\text { Mean age I/C: } 62.9 \pm 8.1 / 63.9 \pm 7.8 \text { years } \\
\text { Gender }(n=\mathrm{M} / \mathrm{F}): 89 / 64 \\
\text { Setting: outpatients } \\
\text { Mean } \% \text { predicted } \mathrm{FEV} \mathrm{V}_{1} \mathrm{I} / \mathrm{C}=49.6 \pm 14.2 / 50.5 \pm 17.0 \\
\text { Mean (SD)dyspnea score } \mathrm{I} / \mathrm{C}=2.2 \pm 1.0 / 2.5 \pm 1.1 \\
\text { COPD exacerbation } n(\%) \mathrm{I} / \mathrm{C}=\text { Not reported }\end{array}$ & 24 months & $\begin{array}{l}\text { Self-management } \\
\text { sessions }\end{array}$ & Not described & $\begin{array}{l}\text { HRQOL Anxiety } \\
\text { and depression }\end{array}$ & $\begin{array}{l}\text { No improvement } \\
\text { in the quality of } \\
\text { life and in the level } \\
\text { of anxiety and } \\
\text { depression. }\end{array}$ \\
\hline $\begin{array}{c}\text { Taylor et al. (2012) } \\
\text { [24] }\end{array}$ & $\begin{array}{l}\text { Better Living with Long-term Airways Disease (BELLA) } \\
\text { delivered by trained two lay tutors. } \\
\text { Intervention includes the following: Manualized, a } 3 \\
\mathrm{~h} \\
\text { session once a week, for seven weeks, at a local } \\
\text { Community center. A session includes COPD } \\
\text { knowledge, maintenance of action plans, skills for } \\
\text { self-management and COPD medications and } \\
\text { counseling }\end{array}$ & $\begin{array}{c}\text { Mean age } \mathrm{I} / \mathrm{C}: 69.0 / 70.5 \text { years } \\
\text { Gender }(n=\mathrm{M} / \mathrm{F}): 78 / 38 \\
\text { Setting: outpatients } \\
\text { Mean \% predicted FEV } \mathrm{FEV}_{1} \mathrm{I} / \mathrm{C}=53.9 \pm 22.6 / 54.6 \pm 23.4 \\
\text { Mean (SD)dyspnea score } \mathrm{I} / \mathrm{C}=\text { Not reported } \\
\text { COPD exacerbation } n(\%) \mathrm{I} / \mathrm{C}=60(77) / 26(68)\end{array}$ & Six months & $\begin{array}{l}\text { Education on } \\
\text { COPD and } \\
\text { self-management }\end{array}$ & Counseling & $\begin{array}{l}\text { HRQOL, Health } \\
\text { status, self-efficacy, } \\
\text { anxiety, } \\
\text { depression, }\end{array}$ & $\begin{array}{l}\text { Improvement in } \\
\text { QOL } \\
\text { (EQ-5D)Improvemen } \\
\text { in self-efficiency } \\
\text { Improvement in } \\
\text { anxiety. }\end{array}$ \\
\hline $\begin{array}{l}\text { Wood-Baker et al. } \\
\text { (2012) [25] }\end{array}$ & $\begin{array}{l}\text { Mentoring by Community Health Nurses. } \\
\text { Intervention includes the following: home visits and } \\
\text { telephone coaching, maintenance of patient diary that } \\
\text { recorded breathlessness, cough, sputum, wellness, } \\
\text { physical activity, and use of reliever medication, along } \\
\text { with monthly reflective feedback meetings }\end{array}$ & $\begin{array}{c}\text { Mean age } \mathrm{I} / \mathrm{C}: 66.5 \pm 9.5 / 69.7 \pm 9.4 \text { years } \\
\text { Gender }(n=\mathrm{M} / \mathrm{F}): 46 / 60 \\
\text { Setting: Inpatients } \\
\text { Mean \% predicted } \mathrm{FEV} 1 \mathrm{I} / \mathrm{C}=34.9 \pm 14.2 / 33.8 \pm 13.6 \\
\text { Mean (SD) dyspnea score } \mathrm{I} / \mathrm{C}=3.4 \pm 1.1 / 3.7 \pm 1.1 \\
\text { COPD exacerbation } n(\%) \mathrm{I} / \mathrm{C}=\text { Not reported }\end{array}$ & 12 months & $\begin{array}{l}\text { Education on } \\
\text { COPD and } \\
\text { self-management } \\
\text { skills }\end{array}$ & $\begin{array}{l}\text { Mentoring to } \\
\text { discuss progress } \\
\text { with clients }\end{array}$ & $\begin{array}{l}\text { HRQOL, anxiety } \\
\text { and depression, } \\
\text { dyspnea and } \\
\text { self-efficacy }\end{array}$ & $\begin{array}{l}\text { Improvement in } \\
\text { physical } \\
\text { functioning of } \\
\text { SF-36 scale } \\
\text { No improvement } \\
\text { in anxiety, } \\
\text { depression, } \\
\text { dyspnea, and } \\
\text { self-efficacy. }\end{array}$ \\
\hline $\begin{array}{l}\text { Bucknall et al. } \\
\text { (2012) [26] }\end{array}$ & $\begin{array}{l}\text { Supported self-management by nurses. } \\
\text { Intervention includes the following: Participants } \\
\text { received four } 40 \text {-min individual training sessions } \\
\text { at home from a study nurse, fortnightly over two } \\
\text { months, with further home visits at least every six } \\
\text { weeks. }\end{array}$ & $\begin{array}{c}\text { Mean age } \mathrm{I} / \mathrm{C}: 70.0 \pm 9.3 / 68.3 \pm 9.2 \text { years } \\
\text { Gender }(n=\mathrm{M} / \mathrm{F}): 170 / 294 \\
\text { Setting: outpatients } \\
\text { Mean \% predicted } \mathrm{FV} \mathrm{V}_{1} \mathrm{I} / \mathrm{C}=41.2 \pm 13.4 / 39.8 \pm 13.8 \\
\text { Mean }(\mathrm{SD}) \text { dyspnea score } \mathrm{I} / \mathrm{C}=\text { Not reported } \\
\text { COPD exacerbation } n(\%) \mathrm{I} / \mathrm{C}=\text { Not reported }\end{array}$ & 12 months & $\begin{array}{l}\text { Education on } \\
\text { COPD, respiratory } \\
\text { drugs, and } \\
\text { self-management } \\
\text { skills }\end{array}$ & $\begin{array}{l}\text { Improving } \\
\text { patients' } \\
\text { confidence }\end{array}$ & $\begin{array}{l}\text { HRQOL, } \\
\text { anxiety and } \\
\text { depression, } \\
\text { self-efficacy }\end{array}$ & $\begin{array}{l}\text { Clinically relevant } \\
\text { improvement in } \\
\text { SGRQ } \\
\text { No improvement } \\
\text { in anxiety and } \\
\text { depression and } \\
\text { self-efficacy. }\end{array}$ \\
\hline
\end{tabular}


Table 1. Cont.

\begin{tabular}{|c|c|c|c|c|c|c|c|}
\hline Study ID & Intervention & Participants Characteristics & Follow-Up & $\begin{array}{c}\text { Activities } \\
\text { Targeted at } \\
\text { Health Literacy }\end{array}$ & $\begin{array}{c}\text { Activities } \\
\text { Targeted to } \\
\text { Activate Patients } \\
\text { in any Form }\end{array}$ & Outcomes & Findings \\
\hline $\begin{array}{c}\text { Fan Vc et al. (2012) } \\
\text { [27] }\end{array}$ & $\begin{array}{l}\text { Intervention delivered by primary care providers } \\
\text { Intervention includes the following: The } \\
\text { Comprehensive Care Management Program (CCMP) } \\
\text { included COPD education during four individual } \\
\text { sessions and one group session, an action plan for } \\
\text { identificication and treatment of exacerbations, and } \\
\text { scheduled a proactive telephone } \\
\text { call for case management. }\end{array}$ & $\begin{array}{c}\text { Mean age } \mathrm{I} / \mathrm{C}: 66.2 \pm 8.4 / 65.8 \pm 8.2 \text { years } \\
\text { Gender }(n=\mathrm{M} / \mathrm{F}): 209 / 204 \\
\text { Setting: outpatients } \\
\text { Mean \% predicted } \mathrm{FEV} \mathrm{V}_{1} \mathrm{I} / \mathrm{C}=38.2 \pm 14.3 / 37.8 \pm 14.5 \\
\text { Mean (SD) dyspnea score } \mathrm{I} / \mathrm{C}=\text { Not reported } \\
\text { COPD exacerbation } n(\%) \mathrm{I} / \mathrm{C}=\text { Not reported }\end{array}$ & 12 months & $\begin{array}{l}\text { Education on } \\
\text { COPD, } \\
\text { medications, and } \\
\text { self-monitoring }\end{array}$ & $\begin{array}{c}\text { Promoting } \\
\text { self-monitoring }\end{array}$ & $\begin{array}{l}\text { HRQOL, and } \\
\text { self-efficacy }\end{array}$ & $\begin{array}{l}\text { No improvement } \\
\text { in health status. } \\
\text { Significant } \\
\text { improvement in } \\
\text { self-efficiency. }\end{array}$ \\
\hline $\begin{array}{l}\text { Bischoff et al. } \\
\text { (2012) [40] }\end{array}$ & $\begin{array}{l}\text { Comprehensive self-management program (CSMP) by } \\
\text { practice nurse (two to four sessions scheduled for } 4-6 \\
\text { weeks). } \\
\text { Intervention includes the following: Paper modules } \\
\text { on COPD disease knowledge, respiratory drugs, } \\
\text { breathing techniques, managing exacerbations, } \\
\text { maintaining a healthy lifestyle, managing stress and } \\
\text { anxiety (optional), and home exercise (optional). }\end{array}$ & $\begin{array}{r}\text { Mean age self }- \text { management, Routine monitoring, and } \\
\text { Usual care: } 65.5 \pm 11.5) / 65.8 \pm 8.3 / 63.5 \pm 10.3 \text { years } \\
\text { Gender: Male: } 37 / 42 / 28 \\
\text { Setting: General Practices } \\
\text { Mean \% predicted FEV } V_{\text {f ror self-management, routine }} \\
\text { monitoring, and usual care }=66.3 \pm 16.5 / 62.9 \pm 14.4 / 67.0 \\
\quad \pm 18.0 \\
\text { Mean (SD) dyspnea score }=2.02 \pm 0.94 / 1.87 \pm 0.72 / 1.73 \\
\quad \pm 0.76 \\
\text { COPD exacerbation }(\text { median, IQ) }=1.0(0-2.0) / 1.0 \\
(0-2.0) /(0.5(0-2.0)\end{array}$ & 18 months & $\begin{array}{l}\text { Education on } \\
\text { COPD, respiratory } \\
\text { drugs, and } \\
\text { self-management } \\
\text { skills }\end{array}$ & Not described & $\begin{array}{l}\text { HRQOL and } \\
\text { Self-efficacy }\end{array}$ & $\begin{array}{l}\text { No improvement } \\
\text { in the quality of } \\
\text { life. } \\
\text { No change in } \\
\text { self-efficacy across } \\
\text { the group. }\end{array}$ \\
\hline $\begin{array}{c}\text { Uijen et al. (2012) } \\
\text { [28] }\end{array}$ & $\begin{array}{l}\text { Dutch translation of the Canadian COPD-specific } \\
\text { self-management program Living Well with COPD } \\
\text { delivered by nurses. } \\
\text { Intervention includes the following: COPD disease } \\
\text { knowledge; use of medication and breathing techniques; } \\
\text { managing exacerbations; maintaining a healthy lifestyle; } \\
\text { managing stress and anxiety; and home exercise. Using } \\
\text { motivational interviewing techniques, the practice } \\
\text { nurses of each practice gave the program to patients in } \\
\text { four individual sessions of } 60 \text { min each. Regular } \\
\text { monitoring: It includes spirometry, inhalation } \\
\text { instructions, and assessment of dyspnea and quality of } \\
\text { life. }\end{array}$ & $\begin{array}{c}\text { Mean age usual/ self-management/regular monitoring: } \\
65.3 \pm 9.3 / 64.3 \pm 11.2 / 63.5 \pm \pm 10.3 \text { years } \\
\text { Gender }(n=\mathrm{M} / \mathrm{F}): 96 / 86 \\
\text { Setting: GP patientsMean } \% \text { predicted FEV }{ }_{1 \text { for }} \\
\text { usual/self-management, Routtine monitoring, and } \\
\text { Usual care }=67.0 \pm 18.0 / 65.8 \pm 16.3 / 67.6 \pm 15.3 \\
\text { Mean }(\mathrm{SD}) \text { dyspnea score }=\text { not reported } \\
\text { COPD exacerbation } n(\%) \mathrm{I} / \mathrm{C}=\text { not reported }\end{array}$ & 24 months & $\begin{array}{l}\text { COPD knowledge } \\
\text { and } \\
\text { self-management } \\
\text { skills }\end{array}$ & $\begin{array}{l}\text { Motivational } \\
\text { interviewing }\end{array}$ & HRQOL & $\begin{array}{l}\text { No significant } \\
\text { improvement in } \\
\text { quality of life. }\end{array}$ \\
\hline $\begin{array}{c}\text { Casey et al. (2013) } \\
\text { [41] }\end{array}$ & $\begin{array}{l}\text { Structured education by nurse and physiotherapists. } \\
\text { Intervention includes the following: Education } \\
\text { sessions. }\end{array}$ & $\begin{array}{c}\text { Mean age I/C: } 68.8 \pm 10.2 \\
/ 68.4 \pm 10.3 \\
\text { Gender }(n=\mathrm{M} / \mathrm{F}): 223 / 127 \\
\text { Setting: General Practices } \\
\text { Mean \% predicted FEV } 1 \mathrm{I} / \mathrm{C}=57.6 \pm 14.3 / 59.7 \pm 13.8 \\
\text { Mean (SD) dyspnea score } \mathrm{I} / \mathrm{C}=\text { Not reported } \\
\text { COPD exacerbation } n(\%) \mathrm{I} / \mathrm{C}=\text { Not reported }\end{array}$ & 15 months & $\begin{array}{l}\text { Education on } \\
\text { COPD and } \\
\text { pulmonary } \\
\text { rehabilitation }\end{array}$ & Not described & $\begin{array}{l}\text { HRQOL } \\
\text { Self-efficacy }\end{array}$ & $\begin{array}{l}\text { No improvement } \\
\text { in total. } \\
\text { No improvement } \\
\text { in self-efficacy. }\end{array}$ \\
\hline
\end{tabular}


Table 1. Cont.

\begin{tabular}{|c|c|c|c|c|c|c|c|}
\hline Study ID & Intervention & Participants Characteristics & Follow-Up & $\begin{array}{c}\text { Activities } \\
\text { Targeted at } \\
\text { Health Literacy }\end{array}$ & $\begin{array}{c}\text { Activities } \\
\text { Targeted to } \\
\text { Activate Patients } \\
\text { in any Form }\end{array}$ & Outcomes & Findings \\
\hline $\begin{array}{l}\text { Mitchell et al. } \\
\text { (2014) [20] }\end{array}$ & $\begin{array}{l}\text { Self-management Program for Activity, Coping, and } \\
\text { Education (SPACE) delivered by the general practitioner } \\
\text { and practice team, including a physiotherapist. } \\
\text { Intervention includes the following: Disease } \\
\text { knowledge, goal setting and coping strategies, exercise } \\
\text { regime, and motivational to enhance new lifestyle } \\
\text { behaviors. }\end{array}$ & $\begin{array}{c}\text { Mean age } \mathrm{I} / \mathrm{C}: 69 \pm 8.0 / 69 \pm 10.1 \text { years } \\
\text { Gender }(n=\mathrm{M} / \mathrm{F}): 89 / 97 \\
\text { Setting: outpatients } \\
\text { Mean \% predicted } \mathrm{FEV} 1 \mathrm{I} / \mathrm{C}=56.0 \pm 16.7 / 59.6 \pm 17.4 \\
\text { Dyspnea grade } 2 / 3 / 4 / 5=(48 / 24 / 13 / 4) /(50 / 22 / 14 / 9) \\
\text { Number of exacerbations in previous } 6 \text { months } \\
\text { 0/1/2/3/4/o5 }=(46 / 31 / 7 / 3 / 1 / 1) /(45 / 33 / 10 / 5 / 1 / 1)\end{array}$ & Six months & $\begin{array}{c}\text { Education on COP } \\
\text { and } \\
\text { self-management }\end{array}$ & $\begin{array}{l}\text { Motivational } \\
\text { interviewing }\end{array}$ & $\begin{array}{c}\text { HRQOL, } \\
\text { self-efficacy, } \\
\text { anxiety, depression }\end{array}$ & $\begin{array}{l}\text { Improvement in } \\
\text { quality of life and } \\
\text { self-efficiency. } \\
\text { No improvement } \\
\text { in anxiety and } \\
\text { depression. } \\
\text { A significant } \\
\text { increase in disease } \\
\text { knowledge. }\end{array}$ \\
\hline $\begin{array}{c}\text { Wang et al. (2014) } \\
\text { [29] }\end{array}$ & $\begin{array}{l}\text { Health Belief Model Nursing } \\
\text { InterventionIntervention includes the } \\
\text { following:Besides the routine nursing care, a 20- to } \\
30 \text {-min HBM based nursing education was } \\
\text { implemented for patients in the intervention group } \\
\text { every two days after their disease conditions were } \\
\text { stable. The tenets of nursing intervention mainly } \\
\text { Included the following: (1) assisting the patients to } \\
\text { perceive the susceptibility and severity of COPD; (2) } \\
\text { assisting them to realize the benefits of the COPD } \\
\text { treatment and the initiation of healthy behaviors in } \\
\text { COPD; (3) assisting them to conquer the obstacles so } \\
\text { that healthy behaviors were applied, and adverse } \\
\text { actions were avoided; (4) improving their confidence in } \\
\text { managing COPD; and (5) alerting them the signals used } \\
\text { to monitor their disease and instructing family members } \\
\text { to support patients for the disease management }\end{array}$ & $\begin{array}{c}\text { Mean age } \mathrm{I} / \mathrm{C}: 71.2(7.4) / 71.9(8.1) \\
\text { Gender }(n=\mathrm{M} / \mathrm{F}): 50 / 38 \\
\text { Setting: Inpatients } \\
\text { Mean (SD) predicted } \mathrm{FEV}_{1} \mathrm{I} / \mathrm{C}=0.7 \pm 0.2 / 8 \pm .8 \pm .4 \\
\text { Mean (SD) dyspnea score } / \mathrm{C}=2.4 \pm 9 / 2.3 \pm .8 \\
\text { COPD exacerbation } n(\%) \mathrm{I} / \mathrm{C}=\text { Not reported }\end{array}$ & Six months & $\begin{array}{l}\text { Education on } \\
\text { COPD, } \\
\text { medications and } \\
\text { self-management } \\
\text { skills }\end{array}$ & $\begin{array}{l}\text { Building } \\
\text { confidence in } \\
\text { managing COPD } \\
\text { with assisting } \\
\text { patients in } \\
\text { perceiving the } \\
\text { susceptibility and } \\
\text { severity of COPD }\end{array}$ & HRQOL & $\begin{array}{c}\text { Improved mean } \\
\text { total scores in the } \\
\text { Health Belief Scale, } \\
\text { except the } \\
\text { perceived disease. }\end{array}$ \\
\hline $\begin{array}{l}\text { Wilson et al. (2015) } \\
\text { [42] }\end{array}$ & $\begin{array}{l}\text { Intervention was delivered by multidisciplinary team. } \\
\text { Intervention includes the following: } 2 \mathrm{~h}(1 \mathrm{~h}) \\
\text { individually tailored exercise training and one-hour } \\
\text { education } \\
\text { program) every three months for one year. }\end{array}$ & $\begin{array}{c}\text { Mean age } \mathrm{I} / \mathrm{C}: 67.3 \pm 15.1 / 69.3 \pm 8.9 \text { years } \\
\text { Gender }(n=\mathrm{M} / \mathrm{F}): 91 / 57 \\
\text { Setting: outpatients } \\
\text { Mean (SD) predicted } \mathrm{FEV} \mathrm{V}_{1} \mathrm{I} / \mathrm{C}=\text { Not reported } \\
\text { Mean (SD) dyspnea score } \mathrm{I} / \mathrm{C}=\text { Not reported } \\
\text { COPD exacerbation } n(\%) \mathrm{I} / \mathrm{C}=\text { Not reported }\end{array}$ & 12 months & $\begin{array}{c}\text { Education on } \\
\text { smoking cessation, } \\
\text { healthy eating, and } \\
\text { the importance of } \\
\text { exercise }\end{array}$ & Not available & $\begin{array}{c}\text { HRQOL } \\
\text { Anxiety and/or } \\
\text { depression }\end{array}$ & $\begin{array}{l}\text { No improvement } \\
\text { in HRQOL. } \\
\text { No improvement } \\
\text { in anxiety and } \\
\text { depression. }\end{array}$ \\
\hline $\begin{array}{c}\text { Kruis et al. (2014) } \\
\text { [30] }\end{array}$ & $\begin{array}{l}\text { Integrated Disease Management (IDM) delivered by } \\
\text { General practitioners, practice nurses, and specialized } \\
\text { physiotherapists. } \\
\text { Intervention includes the following: } \\
\text { action plans, including early recognition and treatment } \\
\text { of exacerbations, encouragement of regular exercise and } \\
\text { guideline based physical reactivation, cooperation with } \\
\text { secondary care, and instructions in nutritional support. }\end{array}$ & $\begin{array}{c}\text { Mean age I/C: } 68.2 \pm 11.3 / 68.4 \pm 1.1 \text { years } \\
\text { Gender }(n=\mathrm{M} / \mathrm{F}): 554 / 532 \\
\text { Setting: outpatients } \\
\text { Mean (SD) predicted } \mathrm{FV} \mathrm{V}_{1} \mathrm{I} / \mathrm{C}=\text { Not reported } \\
\text { Mean }(\mathrm{SD}) \text { dyspea scorea } \mathrm{I} / \mathrm{C}=2.0 \pm 1.3 / 2.0 \pm 1.3 \\
\text { COPD exacerbation } n(\%) \mathrm{I} / \mathrm{C}=\text { Not reported }\end{array}$ & 24 months & $\begin{array}{c}\text { Education on } \\
\text { self-management }\end{array}$ & $\begin{array}{l}\text { Motivational } \\
\text { interviewing to } \\
\text { smoking cessation }\end{array}$ & HRQOL & $\begin{array}{l}\text { No change in } \\
\text { HRQOL. }\end{array}$ \\
\hline
\end{tabular}


Table 1. Cont.

\begin{tabular}{|c|c|c|c|c|c|c|c|}
\hline Study ID & Intervention & Participants Characteristics & Follow-Up & $\begin{array}{l}\text { Activities } \\
\text { Targeted at } \\
\text { Health Literacy }\end{array}$ & $\begin{array}{c}\text { Activities } \\
\text { Targeted to } \\
\text { Activate Patients } \\
\text { in any Form }\end{array}$ & Outcomes & Findings \\
\hline $\begin{array}{l}\text { Hernandez et al. } \\
\text { (2015) [31] }\end{array}$ & $\begin{array}{l}\text { Intervention delivered by a primary care team } \\
\text { (physician, nurse, and social worker) } \\
\text { Intervention includes the following: } 2 \mathrm{~h} \mathrm{educational} \\
\text { program followed by the distribution of patient-specific } \\
\text { support material. The intervention consisted of the } \\
\text { following: (a) patient's empowerment for } \\
\text { self-management; (b) an individualized care plan; (c) } \\
\text { access to a call center; and (d) coordination between the } \\
\text { levels of care. }\end{array}$ & $\begin{array}{c}\text { Mean age } \mathrm{I} / \mathrm{C}: 73 \pm 8 / 75 \pm 9 \text { years } \\
\text { Gender }(n=\mathrm{M} / \mathrm{F}): 131 / 24 \\
\text { Setting: outpatients } \\
\text { Predicted } \% \mathrm{FEV}_{1} \mathrm{I} / \mathrm{C}=41(19) / 44(20) \text { Mean }(\mathrm{SD}) \\
\text { dyspnea score } \mathrm{I} / \mathrm{C}=2.7 \pm 1.3 / 2.5 \pm 1.3 \\
\text { COPD exacerbation } n(\%) \mathrm{I} / \mathrm{C}=\text { Not reported }\end{array}$ & 12 & $\begin{array}{l}\text { Educational } \\
\text { training on } \\
\text { knowledge of } \\
\text { diseases and } \\
\text { self-management } \\
\text { skills }\end{array}$ & $\begin{array}{l}\text { Patient } \\
\text { empower-ment } \\
\text { with social } \\
\text { support and } \\
\text { problem-solving } \\
\text { skills. }\end{array}$ & $\begin{array}{l}\text { HRQOL and } \\
\text { depression and } \\
\text { anxiety }\end{array}$ & $\begin{array}{l}\text { Improved in } \\
\text { health-related } \\
\text { quality of life. } \\
\text { Improvement in } \\
\text { anxiety and } \\
\text { depression. }\end{array}$ \\
\hline $\begin{array}{l}\text { Zwerink et al. } \\
\text { (2016) [4] }\end{array}$ & $\begin{array}{l}\text { Four weekly self-management meetings supervised by } \\
\text { a respiratory nurse and aPhysiotherapist. } \\
\text { Intervention includes the following: } \\
\text { Self-management booklets for patients, patients were } \\
\text { trained in completing daily diaries to record major } \\
\text { symptoms (breathlessness, sputum production, sputum } \\
\text { color) and minor symptoms (cough, wheeze, running } \\
\text { nose, sore throat, fever. Patients also were taught to } \\
\text { recognize the start of an exacerbation, and to initiate a } \\
\text { course of oral prednisolone and/or antibiotics guided by } \\
\text { the action plan. }\end{array}$ & $\begin{array}{c}\text { Mean age } \mathrm{I} / \mathrm{C}: 63.1 \pm 7.9 / 63.7 \pm 8.0 \text { years } \\
\text { Gender }(n=\mathrm{M} / \mathrm{F}): 84 / 50 \\
\text { Setting: outpatients } \\
\text { Mean (SD) predicted FEV } 1 / \mathrm{C}=50.7 \pm 16.3 / 49.6 \pm 15.3 \\
\text { Mean (SD) dyspnea score } \mathrm{I} / \mathrm{C}=2.3 \pm 1.06 / 2.3 \pm 1.14 \\
\text { COPD exacerbation } n(\%) \mathrm{I} / \mathrm{C}=\text { Not reported }\end{array}$ & 24 months & $\begin{array}{l}\text { Education on } \\
\text { self-management } \\
\text { behavior }\end{array}$ & Not described & $\begin{array}{l}\text { HRQOL and, } \\
\text { depression and } \\
\text { anxiety }\end{array}$ & $\begin{array}{l}\text { No improvement } \\
\text { in health-related } \\
\text { quality of life. } \\
\text { No improvement } \\
\text { in anxiety and } \\
\text { depression. }\end{array}$ \\
\hline $\begin{array}{l}\text { Jonsdottir et al. } \\
\text { (2015) [32] }\end{array}$ & $\begin{array}{l}\text { Intervention delivered by lung physician and nurses. } \\
\text { Intervention includes the following: Partnership with } \\
\text { people with COPD and their families with patient- } \\
\text { family conversation in the presence of a trained nurse, } \\
\text { disease information, smoking cessation, and a group } \\
\text { meeting. }\end{array}$ & $\begin{array}{c}\text { Mean age I/C: } 59.4 \pm 4.6 / 58.6 \pm 4.3 \text { years } \\
\text { Gender }(n=\mathrm{M} / \mathrm{F}): 48 / 52 \\
\text { Setting: outpatients }+\mathrm{GP} \text { patients } \\
\text { Mean (SD) predicted } \mathrm{FEV}_{1} \mathrm{I} / \mathrm{C}=\text { Not reported } \\
\text { Mean (SD) dyspnea score } \mathrm{I} / \mathrm{C}=\text { Not reported } \\
\text { COPD exacerbation } n(\%) \mathrm{I} / \mathrm{C}=\text { Not reported }\end{array}$ & Six months & $\begin{array}{l}\text { Education on } \\
\text { COPD, Smoking } \\
\text { cessation and } \\
\text { self-management } \\
\text { skills }\end{array}$ & $\begin{array}{l}\text { Motivation on } \\
\text { specific behavior } \\
\text { like exercise and } \\
\text { nutrition }\end{array}$ & $\begin{array}{l}\text { HRQOL, anxiety, } \\
\text { and depression }\end{array}$ & $\begin{array}{l}\text { No improvement } \\
\text { in QOL and } \\
\text { anxiety and } \\
\text { depression. }\end{array}$ \\
\hline Ko et al. (2017) [7] & $\begin{array}{l}\text { Comprehensive care program delivered by respiratory } \\
\text { nurses. } \\
\text { Intervention includes the following: COPD education } \\
(1 \mathrm{~h} \text { ) and } 3 \text { monthly phone calls to the patients }\end{array}$ & $\begin{array}{c}\text { Mean age I/C: } 74.9 \pm 7.9 / 74.6 \pm 8.6 \\
\text { Gender }(n=\mathrm{M} / \mathrm{F}): 127 / 8 \\
\text { Setting: outpatients } \\
\text { Mean (SD) predicted FEV } \mathrm{I} / / \mathrm{C}=46.7 \pm 18.3 / 44.2 \pm 14.7 \\
\text { Mean (SD) dyspnea score } \mathrm{I} / \mathrm{C}=2 \pm 0.8 / 2.1 \pm 0.8 \\
\text { COPD exacerbation } n(\%) / / \mathrm{C}=1.03 \pm 1.67 / 1.38 \pm 1.58\end{array}$ & 12 months & $\begin{array}{l}\text { Education on } \\
\text { COPD }\end{array}$ & Not described & HRQOL & $\begin{array}{l}\text { Improvement in } \\
\text { quality of life. }\end{array}$ \\
\hline
\end{tabular}


Table 1. Cont.

\begin{tabular}{|c|c|c|c|c|c|c|c|}
\hline Study ID & Intervention & Participants Characteristics & Follow-Up & $\begin{array}{l}\text { Activities } \\
\text { Targeted at } \\
\text { Health Literacy }\end{array}$ & $\begin{array}{l}\text { Activities } \\
\text { Targeted to } \\
\text { Activate Patients } \\
\text { in any Form }\end{array}$ & Outcomes & Findings \\
\hline $\begin{array}{l}\text { Poureslami et al. } \\
\text { (2016) [33] }\end{array}$ & $\begin{array}{l}\text { Intervention delivered by laypersons and doctors } \\
\text { Intervention includes the following:Culturally } \\
\text { specific educational interventions - two videos (one lay } \\
\text { and one clinician video) and one pamphletIn the "lay } \\
\text { video," patients role-played a scenario offering opinions } \\
\text { and narratives about COPD self-management in a } 12 \\
\text { min video clip }\end{array}$ & $\begin{array}{c}\text { Age: } \\
(>75 \text { vs. } \leq 76 \text { years }) \\
\text { Gender }(n=\mathrm{M} / \mathrm{F}): 71 / 20 \\
\text { Setting: outpatients } \\
\text { Mean (SD) predicted } \mathrm{FEV} \mathrm{V}_{1} \mathrm{I} / \mathrm{C}=\text { Not reported } \\
\text { Mean (SD) dyspnea score } \mathrm{I} / \mathrm{C}=\text { Not reported } \\
\text { COPD exacerbation } n(\%) \mathrm{I} / \mathrm{C}=\text { Not reported } \\
\end{array}$ & Nine months & $\begin{array}{c}\text { Educational } \\
\text { materials }\end{array}$ & $\begin{array}{l}\text { Developing the } \\
\text { confidence to use } \\
\text { medication and } \\
\text { recognize } \\
\text { exacerbations to } \\
\text { act correctly) }\end{array}$ & Self-efficacy & $\begin{array}{l}\text { Improvements in } \\
\text { the self-efficacy of } \\
\text { intervention group } \\
\text { participants } \\
\text { relative to the } \\
\text { control group. }\end{array}$ \\
\hline $\begin{array}{c}\text { Ng et al. (2017) } \\
\text { [44] }\end{array}$ & $\begin{array}{l}\text { Intervention delivered by principal investigator and } \\
\text { nurses. } \\
\text { Intervention includes the following:(1) } \\
\text { Self-management education workshops, (2) a patient } \\
\text { handbook and (3) a monthly telephone follow-up. }\end{array}$ & $\begin{array}{c}\text { Mean age } \mathrm{I} / \mathrm{C} \text { : Not provided separately } \\
\text { Gender }(n=\mathrm{M} / \mathrm{F}) \text { : Not provided separately } \\
\text { Setting: outpatients } \\
\text { Mean (SD) predicted FEV } \mathrm{I} / \mathrm{C}=\text { Not reported } \\
\text { Mean (SD) dyspnea score } \mathrm{I} / \mathrm{C}=\text { Not reported } \\
\text { COPD exacerbation } n(\%) \mathrm{I} / \mathrm{C}=\text { Not reported }\end{array}$ & Six months & $\begin{array}{l}\text { Education on } \\
\text { COPD, the natural } \\
\text { course of the } \\
\text { disease, } \\
\text { information on } \\
\text { how to manage a } \\
\text { stable condition, } \\
\text { advice on how to } \\
\text { prevent } \\
\text { complications }\end{array}$ & Not described & Self-efficacy & $\begin{array}{l}\text { Improvement in } \\
\text { self-efficacy. }\end{array}$ \\
\hline $\begin{array}{l}\text { Weldman et al. } \\
\text { (2017) [21] }\end{array}$ & $\begin{array}{l}\text { COPD-GRIP intervention delivered by nurses. } \\
\text { Intervention includes the following: } 4 \text { h educational } \\
\text { session and animation movie was shown. }\end{array}$ & 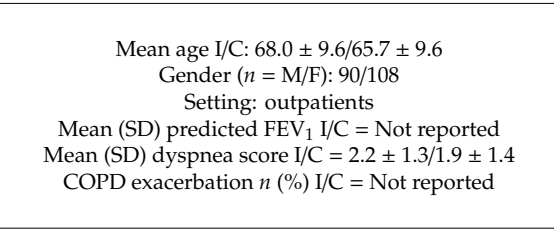 & 12 months & $\begin{array}{l}\text { Education Chronic } \\
\text { Obstructive } \\
\text { Pulmonary } \\
\text { Disease- } \\
\text { Guidance, } \\
\text { Research onIllness } \\
\text { Perception) }\end{array}$ & Not described & $\begin{array}{l}\text { HRQOL } \\
\text { Health education } \\
\text { impact }\end{array}$ & $\begin{array}{l}\text { No significant } \\
\text { differences in } \\
\text { health-related } \\
\text { quality of life. } \\
\text { A significant } \\
\text { change in } \\
\text { health-related } \\
\text { behaviors. }\end{array}$ \\
\hline $\begin{array}{c}\text { Jolly et al. (2018) } \\
\text { [34] }\end{array}$ & $\begin{array}{l}\text { Self-management delivered by a nurse. } \\
\text { Intervention includes the following: Telephone health } \\
\text { coaching delivered by a nurse with supporting written } \\
\text { documents, a pedometer, and a self-monitoring diary } \\
\text { about smoking cessation, physical activity increases, } \\
\text { correct Inhaler use technique, and medication } \\
\text { adherence. }\end{array}$ & $\begin{array}{c}\text { Mean age } \mathrm{I} / \mathrm{C}: 70.7 \pm 8.8 / 70.2 \pm 7.8 \\
\text { Gender }(n=\mathrm{M} / \mathrm{F}): 366 / 211 \\
\text { Setting: outpatients } \\
\text { Mean (SD) predicted FEV } \mathrm{F} / \mathrm{I} / \mathrm{C}=71.2 \pm 18.9 / 72.1 \pm 18.7 \\
\text { Dyspnea } n(\%) \mathrm{I} / \mathrm{C}=89(31) / 76(26) \\
\text { COPD exacerbation } n(\%) \mathrm{I} / \mathrm{C}=\text { Not reported } \\
\end{array}$ & 12 months & $\begin{array}{l}\text { Education on } \\
\text { COPD and } \\
\text { Self-management } \\
\text { behavior }\end{array}$ & $\begin{array}{c}\text { Building patient } \\
\text { confidence in } \\
\text { identifying an } \\
\text { exacerbation early } \\
\text { to start rescue } \\
\text { drugs }\end{array}$ & $\begin{array}{l}\text { HRQOL, } \\
\text { anxiety and } \\
\text { Depression Scale, } \\
\text { self efficacy }\end{array}$ & $\begin{array}{l}\text { No significant } \\
\text { improvement in } \\
\text { health-related } \\
\text { quality of life, } \\
\text { anxiety and } \\
\text { depression, and } \\
\text { self-efficacy. }\end{array}$ \\
\hline $\begin{array}{c}\text { Bringsvor et al. } \\
\text { (2018) [35] }\end{array}$ & $\begin{array}{l}\text { Two moderators and a registered nurse-delivered } \\
\text { intervention and/or physiotherapist. } \\
\text { Intervention includes the following:A salutogenic } \\
\text { orientation was incorporated to improve their } \\
\text { self-management capabilities. Sessions covered were: } \\
\text { problem-solving, goal setting, symptoms, social } \\
\text { challenges, physical activity, nutrition, medication, } \\
\text { smoking cessation, exacerbations, and psychological } \\
\text { issues. }\end{array}$ & $\begin{array}{c}\text { Mean age } \mathrm{I} / \mathrm{C}: \\
68.5 \pm 8.1 / 69.3 \pm 9.0 \\
\text { Gender }(n=\mathrm{M} / \mathrm{F}): 111 / 71 \\
\text { Setting: Community } \\
\text { Mean (SD) predicted FEV } \mathrm{I} / \mathrm{C}=45.2 \pm 14.4 / 44.8 \pm 16.2 \\
\text { Mean (SD) dyspnea score } \mathrm{I} / \mathrm{C}=1.8 \pm 1.0 / 1.7 \pm 1.1 \\
\text { COPD exacerbation } n(\%) \mathrm{I} / \mathrm{C}=\text { Not reported }\end{array}$ & Six months & $\begin{array}{l}\text { Information } \\
\text { booklet was } \\
\text { provided }\end{array}$ & $\begin{array}{l}\text { Salutogenic } \\
\text { approach as } \\
\text { communi-cation } \\
\text { approach }\end{array}$ & $\begin{array}{l}\text { HRQOL, } \\
\text { Sel-efficacy }\end{array}$ & $\begin{array}{l}\text { No significant } \\
\text { improvement in } \\
\text { health-related } \\
\text { quality of life and } \\
\text { self-efficacy. }\end{array}$ \\
\hline
\end{tabular}


Table 1. Cont

\begin{tabular}{|c|c|c|c|c|c|c|c|}
\hline Study ID & Intervention & Participants Characteristics & Follow-Up & $\begin{array}{c}\text { Activities } \\
\text { Targeted at } \\
\text { Health Literacy }\end{array}$ & $\begin{array}{c}\text { Activities } \\
\text { Targeted to } \\
\text { Activate Patients } \\
\text { in any Form }\end{array}$ & Outcomes & Findings \\
\hline $\begin{array}{c}\text { Thom et al. (2018) } \\
\text { [36] }\end{array}$ & $\begin{array}{l}\text { Intervention delivered by pulmonary nurse and } \\
\text { practitioner specialist. } \\
\text { Intervention includes the following: Health coaching } \\
\text { focused on helping patients identify and achieve } \\
\text { self-care goals for their COPD using techniques from } \\
\text { motivational Interviewing and adult learning models. } \\
\text { Specific content included COPD education, action } \\
\text { planning for exacerbations, teaching proper inhaler use, } \\
\text { and facilitating consultation with a pulmonary nurse } \\
\text { practitioner specialist. }\end{array}$ & $\begin{array}{c}\text { Mean age } \mathrm{I} / \mathrm{C}: \\
60.7 \pm 8.0 / 6 \mathrm{C} .9 \pm 7.2 \\
\text { Gender }(n=\mathrm{M} / \mathrm{F}): 126 / 66 \\
\text { Setting: urban public health primary care clinics } \\
\text { Predicted } \% \mathrm{FEV} 1 \mathrm{I} / \mathrm{C}=0.5(0.1) / / 0.6(0.2) \\
\text { Mean (SD) dyspnea score } \mathrm{I} / \mathrm{C}=4.3 \pm 1.4 / 4.6 \pm 1.4 \\
\mathrm{COPD} \text { exacerbation } \\
n(\%) \mathrm{I} / \mathrm{C}=\text { Not reported }\end{array}$ & Nine months & $\begin{array}{l}\text { COPD education } \\
\text { and teaching } \\
\text { about the proper } \\
\text { use of an inhaler. }\end{array}$ & $\begin{array}{l}\text { Motivational } \\
\text { interviewing }\end{array}$ & $\begin{array}{c}\text { HRQOL, } \\
\text { depressive } \\
\text { symptoms, and } \\
\text { self-efficacy }\end{array}$ & $\begin{array}{l}\text { No significant } \\
\text { improvement in } \\
\text { health-related } \\
\text { quality of life, } \\
\text { depression, and } \\
\text { self-efficacy. }\end{array}$ \\
\hline $\begin{array}{l}\text { Steurer-Stey et al. } \\
\quad(2018)[37]\end{array}$ & $\begin{array}{l}\text { "Living well with COPD" COPD self-management } \\
\text { program based on the Chronic Care Model. } \\
\text { Intervention includes the following: Program } \\
\text { consisted of six group modules, including (1) what is } \\
\text { COPD; (2) pharmacological treatment and correct } \\
\text { inhalation techniques; (3) breathing techniques and } \\
\text { coping strategies aimed at symptom control; (4) how to } \\
\text { manage daily activities/energy conservation; (5) the } \\
\text { health benefits of physical activity and how to } \\
\text { determine barriers and enablers of regular physical } \\
\text { activity; and (6) what is an exacerbation and how to } \\
\text { prevent, recognize and adequately manage worsening } \\
\text { symptoms. Special attention was focused on “red flag" } \\
\text { symptoms, like chest pain and/or acute severe dyspnea. }\end{array}$ & $\begin{array}{c}\text { Mean age } \mathrm{I} / \mathrm{C}: \\
69.3 \pm 10.3 / 67.1 \pm 10.0 \\
\text { Gender }(n=\mathrm{M} / \mathrm{F}): 253 / 214 \\
\text { Setting: urban public health primary care clinics } \\
\text { Mean (SD) predicted } \mathrm{FEV} \mathrm{V}_{1} / \mathrm{C}=52.4 \pm 17.6 / 55.9 \pm 16.5 \\
\text { Mean (SD) dyspnea score } \mathrm{I} / \mathrm{C}=4.6 \pm 1.2 / 4.7 \pm 1.6 \\
\text { COPD exacerbation } n(\%) \mathrm{I} / \mathrm{C}=51(71.8) / 88(22.2)\end{array}$ & Two years & $\begin{array}{l}\text { COPD education, } \\
\text { teaching about } \\
\text { proper use of } \\
\text { inhaler and other } \\
\text { self-management } \\
\text { techniques }\end{array}$ & $\begin{array}{l}\text { Motivational } \\
\text { communication } \\
\text { and interviewing }\end{array}$ & $\begin{array}{l}\text { HRQOL and } \\
\text { self-efficacy }\end{array}$ & $\begin{array}{l}\text { Significant } \\
\text { improvement in } \\
\text { health-related } \\
\text { quality of life and } \\
\text { self-efficacy. }\end{array}$ \\
\hline $\begin{array}{l}\text { Aboumatar et al. } \\
\text { (2019) [43] }\end{array}$ & $\begin{array}{l}\text { Intervention was delivered by COPD nurses. } \\
\text { Intervention includes the following:IIndividualized } \\
\text { COPD self-management support to help patients take } \\
\text { medications correctly, recognize exacerbations signs and } \\
\text { follow action plan practice breathing exercises and } \\
\text { energy conservation techniques, maintain an active } \\
\text { lifestyle, seek help as needed, and stop smoking. }\end{array}$ & $\begin{array}{c}\text { Mean age I/C: } 63.9 \pm 9.6 / 66.0 \pm 10.0 \\
\text { Gender }(n=\mathrm{M} / \mathrm{F}):=2 / 148 \\
\text { Setting: outpatients } \\
\text { Mean (SD) predicted FEV } \mathrm{F} / / \mathrm{C}=35.8 \pm 14.2 / 33.3 \pm 16.0 \\
\text { Mean (SD) dyspnea score } \mathrm{I} / \mathrm{C}=\text { Not reported } \\
\text { COPD exacerbation } \\
n(\%) \mathrm{I} / \mathrm{C}=\text { Not reported }\end{array}$ & Six months & $\begin{array}{c}\text { COPD } \\
\text { self-management } \\
\text { education }\end{array}$ & Not described & HRQOL & $\begin{array}{l}\text { No significant } \\
\text { improvement in } \\
\text { health-related } \\
\text { quality of life. }\end{array}$ \\
\hline $\begin{array}{l}\text { Lian Hong et al. } \\
\text { (2019) [38] }\end{array}$ & $\begin{array}{l}\text { A nurse-led self-management program. } \\
\text { Intervention includes the following: Every } \\
\text { participant received five to six face--otoface, individually } \\
\text { tailored education sessions before discharge. The topics } \\
\text { included were: (1) what is COPD and what is its impact; } \\
\text { (2) respiratory muscle training (pursed-lip breathing } \\
\text { and abdominal breathing); (3) medication and } \\
\text { appropriate use of inhalation devices; (4) coughing } \\
\text { techniques; (5) non-pharmacologic strategies for } \\
\text { controlling symptoms; (6) understanding the } \\
\text { importance of physical activities for COPD and how to } \\
\text { choose the right type of exercise; (7) smoking cessation } \\
\text { (if needed); and (8) long-term home oxygen therapy (if } \\
\text { needed). }\end{array}$ & $\begin{array}{c}\text { Mean age } \mathrm{I} / \mathrm{C}: 68.7 \pm 6.2 / 69.2 \pm 6.1 \\
\text { Gender }(n=\mathrm{M} / \mathrm{F}): 121 / 33 \\
\text { Setting: outpatients } \\
\text { Mean (SD) predicted } \mathrm{FEV} \mathrm{V}_{1} \mathrm{I} / \mathrm{C}=58.4 \pm 17.3 / 59.2 \pm 18.2 \\
\text { Mean (SD) dyspnea score } \mathrm{I} / \mathrm{C}=\text { Not reported } \\
\text { COPD exacerbation } \\
n(\%) \mathrm{I} / \mathrm{C}=\text { Not reported }\end{array}$ & 12 months & $\begin{array}{l}\text { Individually } \\
\text { tailored education } \\
\text { sessions }\end{array}$ & $\begin{array}{l}\text { Encourage-ment } \\
\text { and reinforcement }\end{array}$ & HRQOL & $\begin{array}{l}\text { A significant } \\
\text { improvement in } \\
\text { health-related } \\
\text { quality of life. }\end{array}$ \\
\hline
\end{tabular}


Table 1. Cont.

\begin{tabular}{|c|c|c|c|c|c|c|c|}
\hline Study ID & Intervention & Participants Characteristics & Follow-Up & $\begin{array}{c}\text { Activities } \\
\text { Targeted at } \\
\text { Health Literacy }\end{array}$ & $\begin{array}{c}\text { Activities } \\
\text { Targeted to } \\
\text { Activate Patients } \\
\text { in any Form }\end{array}$ & Outcomes & Findings \\
\hline $\begin{array}{l}\text { Ferrone et al. } \\
\text { (2019) [22] }\end{array}$ & $\begin{array}{l}\text { Intervention was provided by a certified respiratory } \\
\text { educator and physician, or usual physician care. } \\
\text { Intervention includes the following: Case } \\
\text { management, education, and skills training, including } \\
\text { self-management education. }\end{array}$ & $\begin{array}{c}\text { Mean age I/C: } 68.6 \pm 9.6 / 67.9 \pm 9.8 \\
\text { Gender }(n=\mathrm{M} / \mathrm{F}): 78 / 90 \\
\text { Setting: outpatients } \\
\text { Mean (SD) predicted } \mathrm{FEV} \mathrm{V}_{1} \mathrm{I} / \mathrm{C}=53.6 \pm 14.2 / 52.0 \pm 14.7 \\
\text { Mean (SD) dyspnea } 5 \text { score } \mathrm{I} / \mathrm{C}=\text { Not reported } \\
\text { COPD exacerbation } n(\%) \mathrm{I} / \mathrm{C}=63(75.0) / 63(75.0)\end{array}$ & 12 months & $\begin{array}{l}\text { Educational } \\
\text { sessions and skills } \\
\text { training }\end{array}$ & $\begin{array}{c}\text { Patients } \\
\text { counseling aimed } \\
\text { at developing } \\
\text { confidence among } \\
\text { patients in various } \\
\text { aspects of } \\
\text { self-management } \\
\text { skills. }\end{array}$ & $\begin{array}{l}\text { HRQOL and } \\
\text { Bristol Knowledge } \\
\text { question-naire }\end{array}$ & $\begin{array}{l}\text { A significant } \\
\text { improvement in } \\
\text { health-related } \\
\text { quality of life and } \\
\text { disease } \\
\text { knowledge. }\end{array}$ \\
\hline
\end{tabular}


Of fifteen studies that addressed self-efficacy, only six showed a positive effect on self-efficacy for behavior changes, such as quitting smoking, performing a daily exercise, or taking medicine according to guidelines. Available literature [9] suggests that increased patient engagements with proper confidence-building may help the patients to maintain health behaviors, and this in turn can improve health outcome. The use of interventions which develop patient activation rather than those which focus on specific behaviors (self-efficacy) may be more useful. This may include tailoring approaches to care based on the levels of patient activation. For example, motivational coaching, along with problem-solving skills and social support, targeted for individuals with low activation levels may help them to understand, carry out, and maintain their role in self-managing their conditions over time [9,47]. We found only two studies [24,31] have showed the improvement in depression and anxiety scores. None of the included studies in our review mentioned any defined actions to address depression and anxiety in the SMI. Therefore, this finding of the review should be interpreted with caution.

Self-management programs need to be guided by learning and behavior-change theories that can be tailored to a population's needs, taking into account literacy, confidence level, ethnic, cultural, and cognitive factors [48]. Interventions aimed at delivering health literacy assume constructing skills for understanding the conditions and relevant information can empower patients, while those aimed at patient activation assume encouragement/motivation standalone can bring positive outcomes [49]. Three of the included studies measured the effect of health literacy, while none measured the effect of patient activation on self-management skills among patients with COPD. Emerging scientific evidence suggests that addressing both health literacy and patient activation components in one intervention might result in better adherence to self-management behaviors in COPD patients [50-52]. Motivational and cognitive-behavioral elements and health coaching have been found to be powerful strategies in helping the patient to become a "successful self-manager" $[37,53,54]$. Thus, the clear understanding between HL and PA, as well as their independent roles and benefits, could help in achieving effective self-management of COPD.

An inherent limitation of this review was the lack of meta-analysis because the intervention and outcome measurements were too heterogeneous. Although multiple databases were searched, using $\mathrm{MeSH}$ terms, the search may not have yielded all published relevant studies, given the variation in terminology for "self-management", "health literacy", and "patient activation".

\section{Conclusions}

This review provided insights into how frequently SMI includes features that address health literacy and patient activation. HL interventions were not comprehensive (largely confided to health education) and PA interventions with improving self-efficacy. This suggests the need to further evaluate the impact of comprehensive self-management interventions, which include elements which address both health literacy and patient activation on health outcomes for COPD patients.

Author Contributions: Conceptualization of the study, U.N.Y.; data selection and extraction, U.N.Y.; analysis, U.N.Y., J.L., and M.F.H.; writing-original draft preparation, U.N.Y., M.F.H., J.L., H.H. and K.P.B.; review and editing, M.F.H. and J.L.; supervision, M.F.H., J.L., H.H. and K.P.B. All authors have read and agreed to the published version of the manuscript.

Funding: This research received no external funding.

Acknowledgments: U.N.Y. is recipient of the International Postgraduate Scholarship (UNSW) and CPHCE (Top-Up Scholarship) for his doctor degree, with which the present review was completed.

Conflicts of Interest: The authors declare no conflict of interest. 


\section{Abbreviations}

$\begin{array}{ll}\text { CCMP } & \text { Comprehensive Care Management Program } \\ \text { COPD } & \text { Chronic Obstructive Pulmonary Diseases } \\ \text { DMP } & \text { Disease Management Program } \\ \text { C } & \text { Control } \\ \text { F } & \text { Females } \\ \text { HRQOL } & \text { Health-related quality of life } \\ \text { HL } & \text { Health Literacy } \\ \text { IDM } & \text { Integrated disease management } \\ \text { I } & \text { Intervention } \\ \text { M } & \text { Males } \\ \text { PSMP } & \text { Partnership based Self-management Training } \\ \text { PA } & \text { Patient Activation } \\ \text { QOL } & \text { Quality of life } \\ \text { SSM } & \text { Supported Self-Management } \\ \text { SMI } & \text { Self-management Intervention }\end{array}$

\section{References}

1. Vestbo, J.; Hurd, S.S.; Agusti, A.G.; Jones, P.W.; Vogelmeier, C.; Anzueto, A.; Barnes, P.J.; Fabbri, L.M.; Martinez, F.J.; Nishimura, M.; et al. Global strategy for the diagnosis, management, and prevention of chronic obstructive pulmonary disease: GOLD executive summary. Am. J. Respir. Crit. Care Med. 2013, 187, 347-365. [CrossRef]

2. Guarascio, A.J.; Ray, S.M.; Finch, C.K.; Self, T.H. The clinical and economic burden of chronic obstructive pulmonary disease in the USA. Clinicoecon. Outcomes. Res. 2013, 5, 235-245. [PubMed]

3. Zwerink, M.; Brusse-Keizer, M.; van der Valk, P.D.; Zielhuis, G.A.; Monninkhof, E.M.; van der Palen, J.; Firth, P.A.; Effing, T. Self-management for patients with chronic obstructive pulmonary disease. Cochrane Database Syst. Rev. 2014, Cd002990. [CrossRef] [PubMed]

4. Zwerink, M.; Kerstjens, H.A.; van der Palen, J.; van der Valk, P.; Brusse-Keizer, M.; Zielhuis, G.; Effing, T. (Cost-)effectiveness of self-treatment of exacerbations in patients with COPD: 2 years follow-up of a RCT. Respirology 2016, 21, 497-503. [CrossRef] [PubMed]

5. Kuzma, A.M.; Meli, Y.; Meldrum, C.; Jellen, P.; Butler-Lebair, M.; Koczen-Doyle, D.; Rising, P.; Stavrolakes, K.; Brogan, F. Multidisciplinary care of the patient with chronic obstructive pulmonary disease. Proc. Am. Thorac. Soc. 2008, 5, 567-571. [CrossRef] [PubMed]

6. Newham, J.J.; Presseau, J.; Heslop-Marshall, K.; Russell, S.; Ogunbayo, O.J.; Netts, P.; Hanratty, B.; Kaner, E. Features of self-management interventions for people with COPD associated with improved health-related quality of life and reduced emergency department visits: A systematic review and meta-analysis. Int. J. Chron. Obstruct. Pulmon. Dis. 2017, 12, 1705-1720. [CrossRef] [PubMed]

7. Ko, F.W.; Cheung, N.K.; Rainer, T.H.; Lum, C.; Wong, I.; Hui, D.S. Comprehensive care programme for patients with chronic obstructive pulmonary disease: A randomised controlled trial. Thorax 2017, 72, 122-128. [CrossRef]

8. Institute of Medicine Committee on Health, L. Health Literacy: A Prescription to End Confusion; Nielsen-Bohlman, L., Panzer, A.M., Kindig, D.A., Eds.; National Academies Press: Washington, DC, USA, 2004.

9. Yadav, U.N.; Hosseinzadeh, H.; Lloyd, J.; Harris, M.F. How health literacy and patient activation play their own unique role in self-management of chronic obstructive pulmonary disease (COPD)? Chron. Respir. Dis. 2019, 16, 1479973118816418. [CrossRef]

10. Nutbeam, D. The evolving concept of health literacy. Soc. Sci. Med. 2008, 67, 2072-2078. [CrossRef]

11. Hibbard, J.H.; Mahoney, E.R.; Stockard, J.; Tusler, M. Development and Testing of a Short Form of the Patient Activation Measure. Health Serv Res. 2005, 40 (6 Pt 1), 1918-1930. [CrossRef]

12. Schaffler, J.; Leung, K.; Tremblay, S.; Merdsoy, L.; Belzile, E.; Lambrou, A.; Lambert, S.D. The Effectiveness of Self-Management Interventions for Individuals with Low Health Literacy and/or Low Income: A Descriptive Systematic Review. J. Gen. Intern. Med. 2018, 33, 510-523. [CrossRef] [PubMed] 
13. Beatty, C.R.; Flynn, L.A.; Costello, T.J. The Impact of Health Literacy Level on Inhaler Technique in Patients with Chronic Obstructive Pulmonary Disease. J. Pharm. Pract. 2017, 30, 25-30. [CrossRef] [PubMed]

14. Jayasinghe, U.W.; Harris, M.F.; Parker, S.M.; Litt, J.; van Driel, M.; Mazza, D.; Del Mar, C.; Lloyd, J.; Smith, J.; Zwar, N. The impact of health literacy and life style risk factors on health-related quality of life of Australian patients. Health Qual. Life Outcomes 2016, 14, 68. [CrossRef] [PubMed]

15. Schroeder, S.A. We Can Do Better-Improving the Health of the American People. N. Engl. J. Med. 2007, 357, 1221-1228. [CrossRef] [PubMed]

16. Bos-Touwen, I.; Schuurmans, M.; Monninkhof, E.M.; Korpershoek, Y.; Spruit-Bentvelzen, L.; Ertugrul-van der Graaf, I.; de wit, N.; Trappenburg, J. Patient and disease characteristics associated with activation for self-management in patients with diabetes, chronic obstructive pulmonary disease, chronic heart failure and chronic renal disease: A cross-sectional survey study. PLoS ONE 2015, 10, e0126400. [CrossRef]

17. Korpershoek, Y.; Bos-Touwen, I.D.; de Man-van Ginkel, J.M.; Lammers, J.W.; Schuurmans, M.J.; Trappenburg, J. Determinants of activation for self-management in patients with COPD. Int. J. Chron. Obstruct. Pulmon. Dis. 2016, 11, 1757-1766. [CrossRef]

18. Liberati, A.; Altman, D.G.; Tetzlaff, J.; Mulrow, C.; Gotzsche, P.C.; Ioannidis, J.P.; Clarke, M.; Devereaux, P.J.; Kleijnen, J.; Moher, D. The PRISMA statement for reporting systematic reviews and meta-analyses of studies that evaluate healthcare interventions: Explanation and elaboration. BMJ (Clin. Res. Ed.) 2009, 339, b2700. [CrossRef]

19. Effing, T.W.; Vercoulen, J.H.; Bourbeau, J.; Trappenburg, J.; Lenferink, A.; Cafarella, P.; Coultas, D.; Meek, P.; Van der Valk, P.; Bischoff, E.W. Definition of a COPD self-management intervention: International Expert Group consensus. Eur. Respir. J. 2016, 48, 46-54. [CrossRef]

20. Mitchell, K.E.; Johnson-Warrington, V.; Apps, L.D.; Bankart, J.; Sewell, L.; Williams, J.E.; Rees, K.; Jolly, K.; Steiner, M.; Morgan, M.; et al. A self-management programme for COPD: A randomised controlled trial. Eur. Respir. J. 2014, 44, 1538-1547. [CrossRef]

21. Weldam, S.W.M.; Schuurmans, M.J.; Zanen, P.; Heijmans, M.; Sachs, A.P.E.; Lammers, J.J. The effectiveness of a nurse-led illness perception intervention in COPD patients: A cluster randomised trial in primary care. ERJ Open Res. 2017, 3, 00115-02016. [CrossRef]

22. Ferrone, M.; Masciantonio, M.G.; Malus, N.; Stitt, L.; O'Callahan, T.; Roberts, Z.; Johnson, L.; Samson, J.; Durocher, L.; Ferrari, M.; et al. The impact of integrated disease management in high-risk COPD patients in primary care. NPJ Prim. Care Respir. Med. 2019, 29, 8. [CrossRef]

23. Chavannes, N.H.; Grijsen, M.; van den Akker, M.; Schepers, H.; Nijdam, M.; Tiep, B.; Muris, J. Integrated disease management improves one-year quality of life in primary care COPD patients: A controlled clinical trial. Prim. Care Respir. J. 2009, 18, 171-176. [CrossRef] [PubMed]

24. Taylor, S.J.C.; Sohanpal, R.; Bremner, S.A.; Devine, A.; McDaid, D.; Fernández, J.L.; Griffiths, C.J.; Eldridge, S. Self-management support for moderate-to-severe chronic obstructive pulmonary disease: A pilot randomised controlled trial. Br. J. Gen. Pract. 2012, 62, e687-e695. [CrossRef] [PubMed]

25. Wood-Baker, R.; Reid, D.; Robinson, A.; Walters, E.H. Clinical trial of community nurse mentoring to improve self-management in patients with chronic obstructive pulmonary disease. Int. J. Chron. Obstruct. Pulmon. Dis. 2012, 7, 407-413. [CrossRef] [PubMed]

26. Bucknall, C.E.; Miller, G.; Lloyd, S.M.; Cleland, J.; McCluskey, S.; Cotton, M.; Stevenson, R.D.; Cotton, P. Glasgow supported self-management trial (GSuST) for patients with moderate to severe COPD: Randomised controlled trial. BMJ (Clin. Res. Ed.) 2012, 344, e1060. [CrossRef]

27. Fan, V.S.; Gaziano, J.M.; Lew, R.; Bourbeau, J.; Adams, S.G.; Leatherman, S.; Thwin, S.S.; Huang, G.D.; Robbins, R.; Sriram, P.S. A comprehensive care management program to prevent chronic obstructive pulmonary disease hospitalizations: A randomized, controlled trial. Ann. Intern. Med. 2012, 156, 673-683. [CrossRef]

28. Uijen, A.A.; Bischoff, E.; Schellevis, F.G.; Bor, H.H.J.; van den Bosch, W.; Schers, H.J. Continuity in different care modes and its relationship to quality of life: A randomised controlled trial in patients with COPD. Br. J. Gen. Pract. 2012, 62, e422-e428. [CrossRef]

29. Wang, Y.; Zang, X.Y.; Bai, J.; Liu, S.Y.; Zhao, Y.; Zhang, Q. Effect of a Health Belief Model-based nursing intervention on Chinese patients with moderate to severe chronic obstructive pulmonary disease: A randomised controlled trial. J. Clin. Nurs. 2014, 23, 1342-1353. [CrossRef] 
30. Kruis, A.L.; Boland, M.R.S.; Assendelft, W.J.J.; Gussekloo, J.; Tsiachristas, A.; Stijnen, T.; Blom, C.; Sont, J.K.; Rutten-Van- Molken, M.P.; Chavannes, N.H. Effectiveness of integrated disease management for primary care chronic obstructive pulmonary disease patients: Results of cluster randomised trial. BMJ Br. Med. J. 2014, 349, g5392. [CrossRef]

31. Hernandez, C.; Alonso, A.; Garcia-Aymerich, J.; Serra, I.; Marti, D.; Rodriguez-Roisin, R.; Narsavage, G.; Gomez, M.C.; Roca, J. Effectiveness of community-based integrated care in frail COPD patients: A randomised controlled trial. NPJ Prim. Care Respir. Med. 2015, 25, 15022. [CrossRef]

32. Jonsdottir, H.; Amundadottir, O.R.; Gudmundsson, G.; Halldorsdottir, B.S.; Hrafnkelsson, B.; Ingadottir, T.S.; Jonsdottir, R.; Jonsson, J.S.; Sigurjonsdottir, E.D.; Stefansdottir, I.K. Effectiveness of a partnership-based self-management programme for patients with mild and moderate chronic obstructive pulmonary disease: A pragmatic randomized controlled trial. J. Adv. Nurs. 2015, 71, 2634-2649. [CrossRef]

33. Poureslami, I.; Kwan, S.; Lam, S.; Khan, N.A.; FitzGerald, J.M. Assessing the effect of culturally specific audiovisual educational interventions on attaining self-management skills for chronic obstructive pulmonary disease in Mandarin- and Cantonese-speaking patients: A randomized controlled trial. Int. J. Chron. Obstruct. Pulmon. Dis. 2016, 11, 1811-1822. [CrossRef] [PubMed]

34. Jolly, K.; Sidhu, M.S.; Hewitt, C.A.; Coventry, P.A.; Daley, A.; Jordan, R.; Heneghan, C.; Singh, S.; Ives, N.; Adab, P.; et al. Self-management of patients with mild COPD in primary care: Randomised controlled trial. BMJ (Clin. Res. Ed.) 2018, 361, k2241. [CrossRef] [PubMed]

35. Bringsvor, H.B.; Langeland, E.; Oftedal, B.F.; Skaug, K.; Assmus, J.; Bentsen, S.B. Effects of a COPD self-management support intervention: A randomized controlled trial. Int. J. Chron. Obstruct. Pulmon. Dis. 2018, 13, 3677-3688. [CrossRef]

36. Thom, D.H.; Willard-Grace, R.; Tsao, S.; Hessler, D.; Huang, B.; DeVore, D.; Chininos, C.; Wolf, J.; Donesky, D.; Garvey, C.; et al. Randomized Controlled Trial of Health Coaching for Vulnerable Patients with Chronic Obstructive Pulmonary Disease. Ann. Am. Thorac. Soc. 2018, 15, 1159-1168. [CrossRef] [PubMed]

37. Steurer-Stey, C.; Dalla Lana, K.; Braun, J.; Ter Riet, G.; Puhan, M.A. Effects of the "Living well with COPD” intervention in primary care: A comparative study. Eur. Respir. J. 2018, 51, 1701375. [CrossRef]

38. Wang, L.H.; Zhao, Y.; Chen, L.Y.; Zhang, L.; Zhang, Y.M. The effect of a nurse-led self-management program on outcomes of patients with chronic obstructive pulmonary disease. Clin. Respir. J. 2019. [CrossRef]

39. Effing, T.; Zielhuis, G.; Kerstjens, H.; van der Valk, P.; van der Palen, J. Community based physiotherapeutic exercise in COPD self-management: A randomised controlled trial. Respir. Med. 2011, 105, 418-426. [CrossRef]

40. Bischoff, E.W.M.A.; Akkermans, R.; Bourbeau, J.; van Weel, C.; Vercoulen, J.H.; Schermer, T.R.J. Comprehensive self management and routine monitoring in chronic obstructive pulmonary disease patients in general practice: Randomised controlled trial. BMJ Br. Med. J. 2012, 345, e7642. [CrossRef]

41. Casey, D.; Murphy, K.; Devane, D.; Cooney, A.; McCarthy, B.; Mee, L.; Newell, J.; O' Shea, E.; Scarrott, C.; Gillespie, P. The effectiveness of a structured education pulmonary rehabilitation programme for improving the health status of people with moderate and severe chronic obstructive pulmonary disease in primary care: The PRINCE cluster randomised trial. Thorax 2013, 68, 922-928. [CrossRef]

42. Wilson, A.M.; Browne, P.; Olive, S.; Clark, A.; Galey, P.; Dix, E.; Woodhouse, H.; Robinson, S.; Wilson, E.C.; Staunton, L. The effects of maintenance schedules following pulmonary rehabilitation in patients with chronic obstructive pulmonary disease: A randomised controlled trial. BMJ Open 2015, 5, e005921. [CrossRef] [PubMed]

43. Aboumatar, H.; Naqibuddin, M.; Chung, S.; Chaudhry, H.; Kim, S.W.; Saunders, J.; Bone, L.; Gurses, A.P.; Knowlton, A.; Pronovost, P.; et al. Effect of a Hospital-Initiated Program Combining Transitional Care and Long-term Self-management Support on Outcomes of Patients Hospitalized With Chronic Obstructive Pulmonary Disease: A Randomized Clinical Trial. JAMA 2019, 322, 1371-1380. [CrossRef] [PubMed]

44. Ng, W.I. Effects of a self-management education program on self-efficacy in patients with COPD: A mixed-methods sequential explanatory designed study. Int. J. Chronic Obstr. Pulm. Dis. 2017, 12, 2129-2139. [CrossRef] [PubMed]

45. Yadav, U.N.; Hosseinzadeh, H.; Baral, K.P. Self-management and patient activation in COPD patients: An evidence summary of randomized controlled trials. Clin. Epidemiol. Glob. Health 2017, 6, 148-154. [CrossRef] 
46. Dineen-Griffin, S.; Garcia-Cardenas, V.; Williams, K.; Benrimoj, S.I. Helping patients help themselves: A systematic review of self-management support strategies in primary health care practice. PLoS ONE 2019, 14, e0220116. [CrossRef] [PubMed]

47. Zimmerman, L.; Wilson, F.A.; Schmaderer, M.S.; Struwe, L.; Pozehl, B.; Paulman, A.; Bratzke, L.C.; Moore, K.; Raetz, L.; George, B. Cost-Effectiveness of a Care Transition Intervention Among Multimorbid Patients. West. J. Nurs. Res. 2017, 39, 622-642. [CrossRef]

48. Chatterjee, S.; Davies, M.J.; Heller, S.; Speight, J.; Snoek, F.J.; Khunti, K. Diabetes structured self-management education programmes: A narrative review and current innovations. Lancet Diabetes Endocrinol. 2018, 6, 130-142. [CrossRef]

49. Schulz, P.J.; Nakamoto, K. Health literacy and patient empowerment in health communication: The importance of separating conjoined twins. Patient Educ. Couns. 2013, 90, 4-11. [CrossRef]

50. Smith, S.G.; Curtis, L.M.; Wardle, J.; von Wagner, C.; Wolf, M.S. Skill Set or Mind Set? Associations between Health Literacy, Patient Activation and Health. PLoS ONE 2013, 8, e74373. [CrossRef]

51. Jacobson, A.F.; Sumodi, V.; Albert, N.M.; Butler, R.S.; DeJohn, L.; Walker, D.; Dion, K.; Tai, H.L.; Ross, D.M. Patient activation, knowledge, and health literacy association with self-management behaviors in persons with heart failure. Heart Lung 2018, 47, 447-451. [CrossRef]

52. Couture, É.M.; Chouinard, M.-C.; Fortin, M.; Hudon, C. The relationship between health literacy and patient activation among frequent users of healthcare services: A cross-sectional study. BMC Fam. Pract. 2018, 19, 38. [CrossRef] [PubMed]

53. Benzo, R.; Vickers, K.; Novotny, P.J.; Tucker, S.; Hoult, J.; Neuenfeldt, P.; Connett, J.; Lorig, K.; McEvoy, C. Health Coaching and Chronic Obstructive Pulmonary Disease Rehospitalization. A Randomized Study. Am. J. Respir. Crit. Care Med. 2016, 194, 672-680. [CrossRef] [PubMed]

54. Spruit, M.A.; Singh, S.J.; Garvey, C.; ZuWallack, R.; Nici, L.; Rochester, C.; Hill, K.; Holland, A.E.; Lareau, S.C.; Man, W.D.; et al. An official American Thoracic Society/European Respiratory Society statement: Key concepts and advances in pulmonary rehabilitation. Am. J. Respir. Crit. Care Med. 2013, 188, e13-e64. [CrossRef] [PubMed]

(C) 2020 by the authors. Licensee MDPI, Basel, Switzerland. This article is an open access article distributed under the terms and conditions of the Creative Commons Attribution (CC BY) license (http://creativecommons.org/licenses/by/4.0/). 